\title{
A LINDSTRÖM THEOREM FOR INTUITIONISTIC PROPOSITIONAL LOGIC
}

\author{
GUILLERMO BADIA AND GRIGORY OLKHOVIKOV
}

\begin{abstract}
It is shown that propositional intuitionistic logic is the maximal (with respect to expressive power) abstract logic satisfying a certain topological property reminiscent of compactness, the Tarski union property and preservation under asimulations.

Keywords: Lindström theorem, intuitionistic logic, abstract model theory, asimulations.

Math subject classification: 03C95, 03B55.
\end{abstract}

\section{INTRODUCTION}

The well-known characterization of classical first-order logic by Per Lindström (published as [15]) has had a great deal of repercussion in contemporary logic. The key development inspired by that paper was the introduction of a notion of "extended first-order logic" that encompassed a great number of expressive extensions of first order logid] which let Lindström establish, roughly, that there were no extensions of classical first order logic that would also satisfy the compactness and Löwenheim-Skolem theorems. A nice accessible exposition can be found in [10].

Lindström's theorem single-handedly started a new area of research known as $a b$ stract or soft model theory (cf. [1, 2]). These two adjectives are used since, when working in this field, one finds themself using "only very general properties of the logic, properties that carry over to a large number of other logics" ([1], p. 225). Some common examples of such properties are compactness, the Craig interpolation theorem or the Beth definability theorem.

Naturally, there is no reason why one could not do non-classical abstract model theory as well, this time focusing on some non-classical logic and its structures. An interesting survey of this much less developed field can be found in [12. The most prominent work has been done in the case of modal logic. A succession of increasingly improved results were obtained by a number of authors such as de Rijke ([6]), van Benthem ([21]), Otto and Piro ([20]) and, more recently, Enqvist ([8]). Indeed, Enqvist's result is a bit more general than the others and it serves as inspiration for this paper. It roughly states that the basic modal language is the most expressive logic (over any class of relational models axiomatizable in first order logic by a collection of strict Horn formulas) satisfying the so called Tarski union property, invariance under bisimilarity and compactness.

\footnotetext{
${ }^{1}$ This was soon replaced by the now more common term "abstract logic". In fact, probably the term "model-theoretic language" ([9]) is more accurate, depending on one's views of what a "logic" is.
} 
The goal of this paper is to obtain a theorem in the vein of Enqvist but for intuitionistic models, an intuitionistic language, replacing invariance under bisimulation for preservation under asimulations and compactness by a topological property more suited to a boolean negationless context. The notion of an asimulation was introduced in $[16]^{2}$ and used to characterize the expressive power of intuitionistic languages as fragments of first order logic over any class of structures axiomatizable by a first order theory. Later the notion of asimulation was modified to capture also expressive powers of intuitionistic first-order logic [17], various systems of basic intuitionistic modal logic [18, and also some systems which are not connected with intuitionistic logic at all [19].

Our work will be of interest not only because it deals with probably the second most famous non-classical logic but because it will be a contribution to abstract model theory without Boolean negation, a subfield where not many results are known.

The layout of the remaining part of the present paper is then as follows. In Section 2 we establish the main bits of our notation and define some basic concepts. Section 3 is then devoted to proving some less immediate lemmas on intuitionistic unravellings and intuitionistically saturated models. In Section 4 we define the notion of an abstract intuitionistic logic and formulate our main result. We prove such result in the next section. In Section [6 we provide topological interpretation of the main theorem. We end the paper by suggesting some lines of further inquiry.

\section{Preliminaries}

In this paper, we consider the language of intuitionistic propositional logic, which we identify with its set of formulas. This language is generated from some set of propositional letters by a finite number of applications of connectives from the set $\{\perp, \top, \wedge, \vee, \rightarrow\}$; the connectives are assumed to have they usual arities. The set of propositional letters can be in general arbitrary large, but we assume that it is disjoint from the above set of connectives (and from the set of logical symbols of every logic which we are going to consider below). Any set with this property we will call vocabulary. Intuitionistic propositional formulas will be denoted with Greek letters like $\varphi$, $\psi$ and $\theta 3^{3}$ whereas the elements of vocabularies will be denoted by letters $p, q$, and $r$. If $\Theta$ is a vocabulary, then $I L(\Theta)$ denotes the set of those intuitionistic propositional formulas which only contain propositional letters from $\Theta$.

For this language, we assume the standard Kripke semantics. The typical notations for intuitionistic Kripke models that we are going to use below are as follows:

$$
\begin{gathered}
\mathcal{M}=\langle W, R, V\rangle, \mathcal{M}^{\prime}=\left\langle W^{\prime}, R^{\prime}, V^{\prime}\right\rangle, \mathcal{M}_{n}=\left\langle W_{n}, R_{n}, V_{n}\right\rangle, \\
\mathcal{N}=\langle U, S, Y\rangle, \mathcal{N}^{\prime}=\left\langle U^{\prime}, S^{\prime}, Y^{\prime}\right\rangle, \mathcal{N}_{n}=\left\langle U_{n}, S_{n}, Y_{n}\right\rangle,
\end{gathered}
$$

where $n \in \omega$. As we proceed, we will also define some operations on intuitionistic Kripke models which are going to affect the notation.

If $\Theta$ is a vocabulary and $\mathcal{M}$ is an intuitionistic Kripke $\Theta$-model, then $W$ is a nonempty set of worlds, states, or nodes, $R$ is a pre-order on $W$ called $\mathcal{M}$ 's accessibility

\footnotetext{
${ }^{2}$ It was an independent rediscovery in somewhat different clothing of the notion of an intuitionistic directed bisimulations from 14 .

${ }^{3}$ We will be adjoining to them subscripts and superscripts when needed, and the same is assumed for any other notations introduced in this paper.
} 
relation, and $V$ is the evaluation function for $\Theta$ in $\mathcal{M}$, that is to say, a function $V: \Theta \rightarrow 2^{W}$ such that for every $p \in \Theta$ and arbitrary $s, t \in W$, it is true that:

$$
w R v \Rightarrow(w \in V(p) \Rightarrow v \in V(p)) .
$$

If $\mathcal{M}$ and $\mathcal{N}$ are two intuitionistic Kripke $\Theta$-models then we say that $\mathcal{M}$ is a submodel of $\mathcal{N}$ and write $\mathcal{M} \subseteq \mathcal{N}$ iff $W \subseteq U, R=S \uparrow(W \times W)$ and, for every $p \in \Theta$, $V(p)=Y(p) \cap W$. In general, for every $W \subseteq U$ there exists a corresponding submodel $\mathcal{M}$ of $\mathcal{N}$ with $W$ as its universe. In such cases we may also denote $\mathcal{M}$ by $\mathcal{N}(W)$.

If $\mathcal{M}_{1} \subseteq, \ldots, \subseteq \mathcal{M}_{n} \subseteq, \ldots$ is a countable chain of intuitionistic Kripke models then the model:

$$
\bigcup_{n \in \omega} \mathcal{M}_{n}=\left(\bigcup_{n \in \omega} W_{n}, \bigcup_{n \in \omega} R_{n}, \bigcup_{n \in \omega} V_{n}\right)
$$

is again an intuitionistic Kripke model.

A pointed intuitionistic Kripke $\Theta$-model is a pair of the form $(\mathcal{M}, w)$ such that $w \in W$. In this paper, we are not going to consider any non-intuitionistic Kripke models. Therefore, we will omit the qualification 'intuitionistic Kripke' in what follows and will simply speak about (pointed) $\Theta$-models. We assume the standard satisfaction relation for $I L(\Theta)$ :

$$
\begin{aligned}
& \mathcal{M}, w \models_{I L} p_{n} \Leftrightarrow w \in V\left(p_{n}\right), \\
& \mathcal{M}, w \models_{I L} \varphi \wedge \psi \Leftrightarrow \mathcal{M}, w \models_{I L} \varphi \text { and } \mathcal{M}, w \models_{I L} \psi ; \\
& \mathcal{M}, w \models_{I L} \varphi \vee \psi \Leftrightarrow \mathcal{M}, w \models_{I L} \varphi \text { or } \mathcal{M}, w \models_{I L} \psi ; \\
& \mathcal{M}, w \models_{I L} \varphi \rightarrow \psi \Leftrightarrow \forall v\left(w R v \Rightarrow\left(\mathcal{M}, v \models_{I L} \varphi \text { or } \mathcal{M}, v \models_{I L} \psi\right)\right) ; \\
& \mathcal{M}, w \models_{I L} \perp ; \\
& \mathcal{M}, w \models_{I L} \top .
\end{aligned}
$$

note that the formulas of $I L(\Theta)$ get satisfied at pointed $\Theta$-models rather than at models alone.

Since classical negation is not available in intuitionistic logic, intuitionistic theories are often defined to include falsehood assumptions along with truth assumptions (this is done in e.g. [11, p. 110] w.r.t. intuitionistic first-order logic). Thus an $I L(\Theta)$-theory becomes a pair $(\Gamma, \Delta) \in 2^{I L(\Theta)} \times 2^{I L(\Theta)}$, where formulas in $\Gamma$ are assumed to be true and formulas from $\Delta$ are assumed to be false. If $(\mathcal{M}, w)$ is a pointed $\Theta$-model, then we define $T h_{I L}(\mathcal{M}, w)$, the $I L(\Theta)$-theory of $(\mathcal{M}, w)$, as follows:

$$
T h_{I L}(\mathcal{M}, w):=\left(\left\{\varphi \in I L(\Theta) \mid \mathcal{M}, w \models_{I L} \varphi\right\},\left\{\varphi \in I L(\Theta) \mid \mathcal{M}, w \nvdash_{I L} \varphi\right\}\right) .
$$

We also introduce a special notation for the left and right projection of $T h_{I L}(\mathcal{M}, w)$, that is to say, for the positive and for the negative part of this theory, denoting them by $T h_{I L}^{+}(\mathcal{M}, w)$ and $T h_{I L}^{-}(\mathcal{M}, w)$, respectively. Inclusion of intuitionistic theories must then involve set-theoretic inclusion of their respective projections, so that we define:

$$
(\Gamma, \Delta) \subseteq\left(\Gamma^{\prime}, \Delta^{\prime}\right) \Leftrightarrow \Gamma \subseteq \Gamma^{\prime} \text { and } \Delta \subseteq \Delta^{\prime} .
$$

It is clear then that an $I L(\Theta)$-theory $(\Gamma, \Delta)$ is $I L$-satisfiable iff we have $(\Gamma, \Delta) \subseteq T h_{I L}(\mathcal{M}, w)$ for some pointed $\Theta$-model $(\mathcal{M}, w)$. In this case we will also write $\mathcal{M}, w \models_{I L}(\Gamma, \Delta)$. If $\mathcal{M} \subseteq \mathcal{N}$ and for every $w \in W$ it is true that $T h_{I L}(\mathcal{M}, w)=T h_{I L}(\mathcal{N}, w)$, then we say that $\mathcal{M}$ is an IL-elementary submodel of $\mathcal{N}$ and write $\mathcal{M} \preccurlyeq I L \mathcal{N}$.

We end this section with some definitions and brief discussion of asimulations and some other related notions relevant to the subject of this paper. 
Definition 1. Let $\left(\mathcal{M}_{1}, w_{1}\right),\left(\mathcal{M}_{2}, w_{2}\right)$ be pointed $\Theta$-models. A binary relation $A$ is called an asimulation from $\left(\mathcal{M}_{1}, w_{1}\right)$ to $\left(\mathcal{M}_{2}, w_{2}\right)$ iff for any $i, j$ such that $\{i, j\}=\{1,2\}$, any $v \in W_{i}, s, t \in W_{j}$, any propositional letter $p \in \Theta$ the following conditions hold:

$$
\begin{array}{ll}
\text { (s-type) } & A \subseteq\left(W_{1} \times W_{2}\right) \cup\left(W_{2} \times W_{1}\right) \\
\text { (elem) } & w_{1} A w_{2} \\
\text { (s-atom) } & \left.\left.\left(v A s \wedge v \in V_{i}(p)\right) \Rightarrow s \in V_{j}(p)\right)\right) \\
\text { (s-back) } & \left(v A s \wedge s R_{j} t\right) \Rightarrow \exists u \in W_{i}\left(v R_{i} u \wedge t A u \wedge u A t\right)
\end{array}
$$

Intuitionistic propositional formulas are known to be preserved under asimulations. More precisely, if $\left(\mathcal{M}_{1}, w_{1}\right)$, and $\left(\mathcal{M}_{2}, w_{2}\right)$ are pointed $\Theta$-models and $A$ is an asimulation from $\left(\mathcal{M}_{1}, w_{1}\right)$ to $\left(\mathcal{M}_{2}, w_{2}\right)$, then $T h_{I L}^{+}\left(\mathcal{M}_{1}, w_{1}\right) \subseteq T h_{I L}^{+}\left(\mathcal{M}_{2}, w_{2}\right)$. Moreover, preservation under asimulations is known to semantically characterize $I L$ as a fragment of classical first-order logic, see [16] for the proof.

Asimulations are defined as an intuitionistic version of bisimulations, a well-known concept from classical modal logic. In fact, bisimulations can be even defined as symmetric asimulations. It follows then that if $B$ is a bisimulation between $\left(\mathcal{M}_{1}, w_{1}\right)$ and $\left(\mathcal{M}_{2}, w_{2}\right)$ then $B$ is an asimulation both from $\left(\mathcal{M}_{1}, w_{1}\right)$ to $\left(\mathcal{M}_{2}, w_{2}\right)$ and from $\left(\mathcal{M}_{2}, w_{2}\right)$ to $\left(\mathcal{M}_{1}, w_{1}\right)$. Further, given a pair of pointed $\Theta$-models $\left(\mathcal{M}_{1}, w_{1}\right)$ and $\left(\mathcal{M}_{2}, w_{2}\right)$ and a bisimulation $B$ between them, we get that $T h_{I L}\left(\mathcal{M}_{1}, w_{1}\right)=T h_{I L}\left(\mathcal{M}_{2}, w_{2}\right)$.

Asimulation is not the only concept from which bisimulation arises as its symmetric version; another (and more traditional) notion of this kind would be simulation which is defined as follows:

Definition 2. Let $\left(\mathcal{M}_{1}, w_{1}\right),\left(\mathcal{M}_{2}, w_{2}\right)$ be pointed $\Theta$-models. A binary relation $Z$ is called an simulation from $\left(\mathcal{M}_{1}, w_{1}\right)$ to $\left(\mathcal{M}_{2}, w_{2}\right)$ iff for any $v, u \in W_{1}, s \in W_{2}$, any propositional letter $p \in \Theta$ the condition elem holds together with the following conditions:

$$
\begin{array}{ll}
\text { (type) } & Z \subseteq\left(W_{1} \times W_{2}\right) \\
\text { (atom) } & \left.\left(v Z s \wedge v \in V_{1}(p)\right) \Rightarrow s \in V_{2}(p)\right) \\
\text { (forth) } & \left(v Z s \wedge v R_{1} u\right) \Rightarrow \exists t \in W_{2}\left(s R_{2} t \wedge u Z t\right)
\end{array}
$$

Note that in the definition of asimulation one finds stronger versions of clauses (type) and (atom), hence the labels (s-type) and (s-atom). The label (s-back) also indicates a strengthening of a more traditional back-clause (given that the symmetry of $A$ is not guaranteed).

Simulations are clearly related to homomorphisms, which we define, a la [3], as follows:

Definition 3. Let $\mathcal{M}_{1}, \mathcal{M}_{2}$ be $\Theta$-models. A function $h: W_{1} \rightarrow W_{2}$ is called a homomorphism from $\mathcal{M}_{1}$ to $\mathcal{M}_{2}$ iff for any $v, u \in W_{1}$, any propositional letter $p \in \Theta$ the following conditions hold:

$\begin{array}{ll}\text { (atom) } & v \in V_{1}(p) \Rightarrow h(v) \in V_{2}(p) \\ \text { (hom) } & v R_{1} u \Rightarrow h(v) R_{2} h(u)\end{array}$

It is clear that the main difference between simulations and homomorphisms is that the latter are functions while the former are relations. We will see in the next section 
that a sort of connection between the two can be established and this fact is important for our result.

A stronger version of asimulation in the context of intuitionistic logic would be an $I L$-embedding defined as follows:

Definition 4. Let $\mathcal{M}_{1}, \mathcal{M}_{2}$ be $\Theta$-models. A function $h: W_{1} \rightarrow W_{2}$ is called an $I L$ embedding of $\mathcal{M}_{1}$ into $\mathcal{M}_{2}$ iff $h$ is an injective homomorphism from $\mathcal{M}_{1}$ to $\mathcal{M}_{2}$, and for all $v, u \in W_{1}$ it is true that

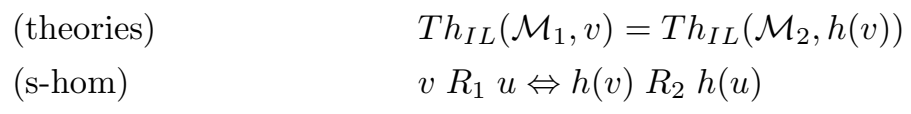

It is easy to notice that whenever $f$ is an $I L$-embedding of $\mathcal{M}_{1}$ into $\mathcal{M}_{2}$, we have that $\mathcal{M}_{2}(f(W)) \preccurlyeq_{I L} \mathcal{M}_{2}$. Also $\mathcal{M}_{1}$ is obviously isomorphic to $\mathcal{M}_{2}(f(W))$, so that replacing $\mathcal{M}_{2}(f(W))$ with a copy of $\mathcal{M}_{1}$ changes nothing. Therefore, whenever there exists is an $I L$-embedding of $\mathcal{M}_{1}$ into $\mathcal{M}_{2}$ we may assume that $\mathcal{M}_{1} \preccurlyeq{ }_{I L} \mathcal{M}_{2}$

\section{INTUITIONISTIC UNRAVELLINGS AND SATURATED MODELS}

In this section, we treat some less immediate properties of intuitionistic logic. We start by introducing a further piece of notation. If $\left(o_{1}, \ldots, o_{n}\right)$ is an $n$-tuple of objects of any nature, then we will denote it with $\bar{o}_{n}$.

Unravelling Kripke models is another well-known item from classical modal logic. Here we adapt it to the intuitionistic setting. For a given intuitionistic pointed Kripke $\Theta$-model $(\mathcal{M}, w)$, the model $\mathcal{M}_{w}^{u n}=\left\langle W_{w}^{u n}, R_{w}^{u n}, V_{w}^{u n}\right\rangle$, called the intuitionistic unravelling of $\mathcal{M}$ around $w$ is defined as follows.

- $W_{w}^{u n}=\left\{\bar{u}_{n} \in W^{n} \mid u_{1}=w,(\forall i<n)\left(w_{i} R w_{i+1}\right)\right\}$

- $R_{w}^{u n}$ is the reflexive and transitive closure of the following relation: $\left\{(s, t) \in W^{2} \mid(\exists u \in W)(t=(s, u))\right\}$;

- For arbitrary $p \in \Theta$, we have $V_{w}^{u n}(p)=\left\{\bar{u}_{n} \in W_{w}^{u n} \mid u_{n} \in V(p)\right\}$.

The following lemma sums up the basic facts about intuitionistic unravellings:

Lemma 1. Let $(\mathcal{M}, w)$ be a pointed $\Theta$-model. Then:

(1) $\mathcal{M}_{w}^{u n}$ is a $\Theta$-model;

(2) $R_{w}^{u n}$ is antisymmetric;

(3) $\left(\mathcal{M}_{w}^{u n}, w\right)$ is bisimilar to $(\mathcal{M}, w)$;

(4) $T h_{I L}\left(\mathcal{M}, v_{k}\right)=T h_{I L}\left(\mathcal{M}, \bar{v}_{k}\right)$ for any $\bar{v}_{k} \in W_{w}^{u n}$;

(5) $T h_{I L}\left(\mathcal{M}, \bar{u}_{n}\right)=T h_{I L}\left(\mathcal{M}, \bar{v}_{k}\right)$ for any $\bar{u}_{n}, \bar{v}_{k} \in W_{w}^{u n}$ whenever $u_{n}=v_{k}$.

Proof. By definition, $R_{w}^{u n}$ is reflexive and transitive. Moreover, we note that for arbitrary $\bar{w}_{k}, \bar{v}_{n} \in W_{w}^{u n}$ we have

$$
\bar{w}_{k} R_{w}^{u n} \bar{v}_{n} \Leftrightarrow k \leq n \wedge \bar{w}_{k}=\bar{v}_{k} .
$$

Therefore, to show antisymmetry, assume that for a given $\bar{w}_{k}, \bar{v}_{n} \in W_{w}^{u n}$ we have

$$
\bar{w}_{k} R_{w}^{u n} \bar{v}_{n} \wedge \bar{v}_{n} R_{w}^{u n} \bar{w}_{k} .
$$

By the above biconditional it immediately follows that $k \leq n \wedge n \leq k$ so that we have $k=n$ and, further, that $\bar{w}_{k}=\bar{v}_{k}$. Therefore, we get $\bar{w}_{k}=\bar{v}_{n}$ and thus $R_{w}^{u n}$ is shown to be antisymmetric. 
To show monotonicity of $V_{w}^{u n}$ w.r.t. $R_{w}^{u n}$, assume that for some $\bar{w}_{k}, \bar{v}_{n} \in W_{w}^{u n}$ we have $\bar{w}_{k} R_{w}^{u n} \bar{v}_{n}$. Then we have both $k \leq n$ and $\bar{w}_{k}=\bar{v}_{k}$, so that, in particular, we get $w_{k}=v_{k}$. Further, by definition of $W_{w}^{u n}$ we have that

$$
v_{k} R v_{k+1} R, \ldots, R v_{n-1} R v_{n},
$$

whence, by monotonicity of $V$ w.r.t. $R$, it follows that for every $p \in \Theta$, if $v_{k} \in V(p)$, then $v_{n} \in V(p)$. It remains to notice that, by definition of $V_{w}^{u n}$ we have both

$$
\bar{w}_{k} \in V_{w}^{u n}(p) \Leftrightarrow w_{k} \in V(p) \Leftrightarrow v_{k} \in V(p)
$$

(by $\left.w_{k}=v_{k}\right)$ and

$$
\bar{v}_{n} \in V_{w}^{u n}(p) \Leftrightarrow v_{n} \in V(p),
$$

whence we obtain that $\bar{v}_{n} \in V_{w}^{u n}(p)$, whenever $\bar{w}_{k} \in V_{w}^{u n}(p)$.

As for the bisimulation $B$ between $\left(\mathcal{M}_{w}^{u n}, w\right)$ and $(\mathcal{M}, w)$, it can be defined as follows:

$$
B:=\left\{\left(w_{n}, \bar{w}_{n}\right),\left(\bar{w}_{n}, w_{n}\right) \mid(\exists k \geq 0)\left(w R_{w}^{u n} w_{n}\right)\right\} .
$$

Parts 4 and 5 then follow from this definition of bisimulation and preservation of intuitionistic formulas under asimulations.

We state here one more lemma on intuitionistic unravellings relevant to our main result:

Lemma 2. Let $(\mathcal{M}, w)$ and $(\mathcal{N}, u)$ be pointed $\Theta$-models, and let $Z$ be a simulation from $\left(\mathcal{M}_{w}^{u n}, w\right)$ to $(\mathcal{N}, u)$ such that the left projection of $Z$ is $W_{w}^{u n}$ (that is to say, $Z$ is total). Then there exists a homomorphism $h: \mathcal{M}_{w}^{u n} \rightarrow \mathcal{N}$ such that $h(w)=u$ and $v Z h(v)$ for every $v \in W_{w}^{u n}$.

Proof. Suppose that there is total simulation $Z$ from $\left(\mathcal{M}_{w}^{u n}, w\right)$ to $(\mathcal{N}, u)$. We then define $h$ by induction on the length of $\bar{v}_{n} \in W_{w}^{u n}$. The base case is when $\bar{v}_{n}=w$ and we set $h(w):=u$. If $n=k+1$, then $h\left(\bar{v}_{k}\right)$ must be already defined by induction hypothesis, and we must also have $\bar{v}_{k} Z h\left(\bar{v}_{k}\right)$. Also, we have $\bar{v}_{k} R_{w}^{u n} \bar{v}_{k+1}$. But then by condition (forth), there must be some $s \in U$ such that $h\left(\bar{v}_{k}\right) Z s$ and $\bar{v}_{k+1} Z s$. Choose one such $s$ and set $h\left(\bar{v}_{k+1}\right):=s$. Since $\bar{v}_{k}$, the immediate predecessor of $\bar{v}_{k+1}$ different from $\bar{v}_{k+1}$ in $\mathcal{M}_{w}^{u n}$ is unique (naturally, there might be other predecessors of $\bar{v}_{k+1}$ but they have to lay below $\bar{v}_{k}$ ), $h$ is a function with the required properties.

Let $(\Gamma, \Delta)$ be an $I L(\Theta)$-theory and $\mathcal{M}$ be a $\Theta$-model. If $v \in W$, we will say that $(\Gamma, \Delta)$ is finitely IL-satisfiable in $\mathcal{M}$ by successors of $v$ iff for all finite $\Gamma^{\prime} \subseteq \Gamma$ and $\Delta^{\prime} \subseteq \Delta$, there is a $u \in W$ such that $v R u$ and $\mathcal{M}, u \models_{I L}\left(\Gamma^{\prime}, \Delta^{\prime}\right)$. A $\Theta$-model $\mathcal{M}$ is called $I L$-saturated iff for every $v \in W$ and for every $I L(\Theta)$-theory $(\Gamma, \Delta)$, whenever $(\Gamma, \Delta)$ is finitely $I L$-satisfiable in $\mathcal{M}$ by successors of $v$, then for some $u \in W$ it is true that $v R u$ and $\mathcal{M}, u \models_{I L}(\Gamma, \Delta)$. The importance of intuitionistically saturated models is that among them asimulations can be defined in the following easy and natural way:

Lemma 3. Let $\left(\mathcal{M}_{1}, w_{1}\right),\left(\mathcal{M}_{2}, w_{2}\right)$ be pointed $\Theta$-models. If $T h_{I L}^{+}\left(\mathcal{M}_{1}, w_{1}\right) \subseteq T h_{I L}^{+}\left(\mathcal{M}_{2}, w_{2}\right)$ and both $\mathcal{M}_{1}$ and $\mathcal{M}_{2}$ are intuitionistically saturated, then the relation $A$ such that for all $u \in W_{i}, s \in W_{j}$ if $\{i, j\}=\{1,2\}$, then

$$
u A s \Leftrightarrow\left(T h_{I L}^{+}\left(\mathcal{M}_{i}, u\right) \subseteq T h_{I L}^{+}\left(\mathcal{M}_{j}, s\right)\right)
$$

is an asimulation from $\left(\mathcal{M}_{1}, w_{1}\right)$ to $\left(\mathcal{M}_{2}, w_{2}\right)$. 
Proof. The relation $A$, as defined in the lemma, obviously satisfies conditions (s-type, (elem), and (s-atom) given in Definition 1. We check the remaining condition, (s-back).

Assume that $u A s$, so that $T h_{I L}^{+}\left(\mathcal{M}_{i}, u\right) \subseteq T h_{I L}^{+}\left(\mathcal{M}_{j}, s\right)$, and let for some $t \in W_{j}$ we have $s R_{j} t$. Let $\left(\Gamma^{\prime}, \Delta^{\prime}\right) \subseteq T h_{I L}\left(\mathcal{M}_{j}, t\right)$ be finite. Then, of course, we have:

$$
\mathcal{M}_{j}, s \forall_{I L} \bigwedge \Gamma^{\prime} \rightarrow \bigvee \Delta^{\prime}
$$

and, by $u A s$ :

$$
\mathcal{M}_{i}, u \not \nvdash_{I L} \bigwedge \Gamma^{\prime} \rightarrow \bigvee \Delta^{\prime}
$$

The latter means that $\left(\Gamma^{\prime}, \Delta^{\prime}\right)$ must be $I L$-satisfied by some $R_{i}$-successor of $u$. Therefore, by intuitionistic saturation of both $\mathcal{M}_{1}$ and $\mathcal{M}_{2}$, we get that for some $v \in W_{i}$ such that $u R_{i} w$ the theory $T h_{I L}\left(\mathcal{M}_{j}, t\right)$ is $I L$-satisfied at $\left(\mathcal{M}_{i}, u\right)$. It follows immediately that

whence

$$
T h_{I L}\left(\mathcal{M}_{i}, w\right)=T h_{I L}\left(\mathcal{M}_{j}, t\right),
$$

$$
T h_{I L}^{+}\left(\mathcal{M}_{i}, w\right)=T h_{I L}^{+}\left(\mathcal{M}_{j}, t\right),
$$

which, in turn, means that both $w A t$ and $t A w$.

\section{Abstract intuitionistic LOGics}

An abstract intuitionistic logic $\mathcal{L}$ is a pair $\left(L, \models_{\mathcal{L}}\right)$, where $L$ maps every vocabulary $\Theta$ to the set $L(\Theta)$ of $\Theta$-formulas of $\mathcal{L}$ and $\models_{\mathcal{L}}$ is a binary relation between pointed models and elements of $L(\Theta)$ for some vocabulary $\Theta$ such that the following conditions are satisfied:

- $\Theta \subseteq \Theta^{\prime} \Rightarrow L(\Theta) \subseteq L\left(\Theta^{\prime}\right)$.

- If $\mathcal{M}$ is a $\Theta$-model and $\mathcal{M}, w \models_{\mathcal{L}} \phi$, then $\phi \in L(\Theta)$.

- If $\mathcal{M}$ and $\mathcal{N}$ are $\Theta$-models, $\phi \in L(\Theta)$ and $f$ is an isomorphism between $\mathcal{M}$ and $\mathcal{N}$, then for every $w \in W$ it is true that:

$$
\mathcal{M}, w \models_{\mathcal{L}} \phi \Leftrightarrow \mathcal{N}, f(w) \models_{\mathcal{L}} \phi .
$$

- (Expansion). If $\Theta$ is a vocabulary, $\phi \in L(\Theta), \Theta \subseteq \Theta^{\prime}, \mathcal{M}$ is a $\Theta^{\prime}$-model, and $\mathcal{M}\lceil\Theta$ is the reduct of $\mathcal{M}$ to $\Theta$, then:

$$
\mathcal{M}, w \models_{\mathcal{L}} \phi \Leftrightarrow \mathcal{M} \uparrow \Theta, w \models_{\mathcal{L}} \phi .
$$

- (Occurrence). If $\phi \in L(\Theta)$ for some vocabulary, then there is a finite $\Theta_{\phi} \subseteq \Theta$ such that for every $\Theta^{\prime}$-model $\mathcal{M}$, the relation $\mathcal{M} \models_{\mathcal{L}} \phi$ is defined iff $\Theta_{\phi} \subseteq \Theta^{\prime}$.

- (Closure). For every vocabulary $\Theta$ and all $\phi, \psi \in L(\Theta)$, we have $\phi \rightarrow \psi, \phi \wedge \psi, \phi \vee \psi \in L(\Theta)$, that is to say, $\mathcal{L}$ is closed under intuitionistic implication, conjunction and disjunction.

We further define that given a pair of abstract intuitionistic logics $\mathcal{L}$ and $\mathcal{L}^{\prime}$, we say that $\mathcal{L}^{\prime}$ extends $\mathcal{L}$ and write $\mathcal{L} \unlhd \mathcal{L}^{\prime}$ when for all vocabularies $\Theta$ and $\phi \in L(\Theta)$ there exists a $\psi \in L^{\prime}(\Theta)$ such that for arbitrary pointed $\Theta$-model $(\mathcal{M}, w)$ it is true that:

$$
\mathcal{M}, w \models_{\mathcal{L}} \phi \Leftrightarrow \mathcal{M}, w \models_{\mathcal{L}^{\prime}} \psi .
$$

If both $\mathcal{L} \unlhd \mathcal{L}^{\prime}$ and $\mathcal{L}^{\prime} \unlhd \mathcal{L}$ holds, then we say that the logics $\mathcal{L}$ and $\mathcal{L}^{\prime}$ are expressively equivalent and write $\mathcal{L} \equiv \mathcal{L}^{\prime}$.

It is easy to see that intuitionistic propositional logic itself turns out to be an abstract intuitionistic logic $\mathrm{IL}=\left(I L, \models_{I L}\right)$ under this definition. It is also obvious that the above definitions and conventions about intuitionistic theories can be carried over to an 
arbitrary abstract intuitionistic logic $\mathcal{L}$ replacing everywhere $I L$ with $\mathcal{L}$, including such notions as elementary submodel, embedding, saturation of a model, etc. In particular, since the relation $\models_{\mathcal{L}}$ never distinguishes between isomorphic models, the remark after Definition 4 holds for arbitrary intuitionistic logics $\mathcal{L}$.

In this paper, our specific interest is in the extensions of IL. Since every abstract intuitionistic logic $\mathcal{L}$ extending IL must have an equivalent for every intuitionistic propositional formula, we will just assume that for every vocabulary $\Theta$ we have $I L(\Theta) \subseteq L(\Theta)$ and that for every $\varphi \in I L(\Theta)$ and every pointed $\Theta$-model $(\mathcal{M}, w)$ we have that:

$$
\mathcal{M}, w \models_{\mathcal{L}} \varphi \Leftrightarrow \mathcal{M}, w \models_{\mathrm{IL}} \varphi,
$$

so that all the intuitionistic propositional formulas are present in $\mathcal{L}$ in their usual form and with their usual meaning, and whatever other formulas that $\mathcal{L}$ may contain are distinct from the elements of $I L(\Theta)$.

We can immediately state the following corollary to Lemma 3 for arbitrary extensions of IL:

Corollary 1. Let IL $\unlhd \mathcal{L}$, and let $\left(\mathcal{M}_{1}, w_{1}\right),\left(\mathcal{M}_{2}, w_{2}\right)$ be two pointed intuitionistic Kripke $\Theta$-models. If $T h_{I L}^{+}\left(\mathcal{M}_{1}, w_{1}\right) \subseteq T h_{I L}^{+}\left(\mathcal{M}_{2}, w_{2}\right)$ and both $\mathcal{M}_{1}$ and $\mathcal{M}_{2}$ are $\mathcal{L}$ saturated, then the relation $A$ such that for all $u \in W_{i}, s \in W_{j}$ if $\{i, j\}=\{1,2\}$, then

$$
u A s \Leftrightarrow\left(T h_{I L}^{+}\left(\mathcal{M}_{i}, u\right) \subseteq T h_{I L}^{+}\left(\mathcal{M}_{j}, s\right)\right)
$$

is an asimulation from $\left(\mathcal{M}_{1}, w_{1}\right)$ to $\left(\mathcal{M}_{2}, w_{2}\right)$.

To prove this, we just repeat the proof of Lemma 3 using the fact that every $\mathcal{L}$ saturated model is of course $I L$-saturated.

Some of the extensions of IL turn out to be better than others in that they have useful model-theoretic properties. We define some of the relevant properties below.

Definition 5. Let $\mathcal{L}=\left(L, \models_{\mathcal{L}}\right)$ be an abstract intuitionistic logic. Then:

- $\mathcal{L}$ is invariant under asimulations, iff for all vocabularies $\Theta$ and arbitrary pointed $\Theta$-models $\left(\mathcal{M}_{1}, w_{1}\right)$ and $\left(\mathcal{M}_{2}, w_{2}\right)$, whenever $A$ is an asimulation from $\left(\mathcal{M}_{1}, w_{1}\right)$ to $\left(\mathcal{M}_{2}, w_{2}\right)$, then the inclusion $\operatorname{Th}_{\mathcal{L}}^{+}\left(\mathcal{M}_{1}, w_{1}\right) \subseteq T h_{\mathcal{L}}^{+}\left(\mathcal{M}_{2}, w_{2}\right)$ holds.

- $\mathcal{L}$ is intuitionistically compact, iff an arbitrary $L(\Theta)$-theory $(\Gamma, \Delta)$ is $\mathcal{L}$ satisfiable, whenever for every finite $\Gamma^{\prime} \subseteq \Gamma$ and $\Delta^{\prime} \subseteq \Delta$, the theory $\left(\Gamma^{\prime}, \Delta^{\prime}\right)$ is $\mathcal{L}$-satisfiable.

- $\mathcal{L}$ has Tarski Union Property (TUP) iff for every $\mathcal{L}$-elementary chain

$$
\mathcal{M}_{0} \preccurlyeq \mathcal{L}, \ldots, \preccurlyeq \mathcal{L} \mathcal{M}_{n} \preccurlyeq \mathcal{L}, \ldots
$$

it is true that:

$$
\mathcal{M}_{n} \preccurlyeq \mathcal{L} \bigcup_{n \in \omega} \mathcal{M}_{n}
$$

for all $n \in \omega$.

We have mentioned above that in the case of IL invariance under asimulations implies invariance under bisimulations. The same argument holds in the case of arbitrary abstract intuitionistic logic $\mathcal{L}$. In other words, the following lemma holds:

Lemma 4. If $\mathcal{L}$ is an abstract intuitionistic logic that is invariant under asimulations, then $\mathcal{L}$ is invariant under bisimulations. In other words, for arbitrary vocabulary $\Theta$ 
and arbitrary pointed $\Theta$-models $\left(\mathcal{M}_{1}, w_{1}\right)$ and $\left(\mathcal{M}_{2}, w_{2}\right)$, if $B$ is bisimulation between $\left(\mathcal{M}_{1}, w_{1}\right)$ and $\left(\mathcal{M}_{2}, w_{2}\right)$, then:

$$
T h_{\mathcal{L}}\left(\mathcal{M}_{1}, w_{1}\right)=T h_{\mathcal{L}}\left(\mathcal{M}_{2}, w_{2}\right) .
$$

Moreover if an abstract intuitionistic logic is invariant under asimulations, then every formula of this logic is monotonic w.r.t. accessibility relation. More precisely, the following lemma holds:

Lemma 5. If $\mathcal{L}$ is an abstract intuitionistic logic that is invariant under asimulations, then for every pointed $\Theta$-model $(\mathcal{M}, w)$, for every $v \in W$ such that $w R v$, and for every $\phi \in L(\Theta)$ it is true that:

$$
\mathcal{M}, w \models_{\mathcal{L}} \phi \Rightarrow \mathcal{M}, v \models_{\mathcal{L}} \phi .
$$

Proof. We define asimulation $A$ from $(\mathcal{M}, w)$ to $(\mathcal{M}, v)$ setting:

$$
A:=\{(w, v)\} \cup\{(u, u) \mid u \in W, v R u\} .
$$

The lemma then follows by asimulation invariance of $\mathcal{L}$.

Note that for Lemma 5 one does not even need to assume that IL $\unlhd \mathcal{L}$. The next lemma sums up some well-known facts about IL:

Lemma 6. IL is invariant under asimulations, intuitionistically compact and has TUP.

Proof. Invariance under asimulations follows from the main result of [16]. The intuitionistic compactness of IL is a well-known fact and is established in many places, see e.g. [4, Theorem 2.46]. The proof of TUP runs along the lines of standard proof of TUP for modal formalisms ( $\mathrm{cf}$ [20, Observation 5]); we briefly sketch it here.

We need to show that if $\varphi \in I L(\Theta), n \in \omega$ and $w \in W_{n}$, then

$$
\mathcal{M}_{n}, w \models_{I L} \varphi \Leftrightarrow \bigcup_{n \in \omega} \mathcal{M}_{n}, w \models_{I L} \varphi ;
$$

this is done by induction on $\varphi$ and the only non-trivial case is when $\varphi=\psi \rightarrow \chi$. If $\mathcal{M}_{n}, w \forall_{I L} \psi \rightarrow \chi$, then there is a $v \in W_{n}$ such that $w R_{n} v$ and $\mathcal{M}_{n}, v \models_{I L}(\{\psi\},\{\chi\})$. But then, by induction hypothesis we must have $\bigcup_{n \in \omega} \mathcal{M}_{n}, v \models_{I L}(\{\psi\},\{\chi\})$ and we also have $w\left(\bigcup_{n \in \omega} R_{n}\right) v$ so that $\bigcup_{n \in \omega} \mathcal{M}_{n}, w \nvdash_{I L} \psi \rightarrow \chi$. In the other direction, assume that $\bigcup_{n \in \omega} \mathcal{M}_{n}, w \forall_{I L} \psi \rightarrow \chi$. Then, for some $v \in \bigcup_{n \in \omega} W_{n}$ such that $w\left(\bigcup_{n \in \omega} R_{n}\right) v$ it is true that $\bigcup_{n \in \omega} \mathcal{M}_{n}, v \models_{I L}(\{\psi\},\{\chi\})$. But then, for some $k \geq n$, we must have both $w, v \in W_{k}$ and $w R_{k} v$, so that we get $\mathcal{M}_{k}, w \nvdash_{I L} \psi \rightarrow \chi$. By obvious transitivity of $\preccurlyeq_{I L}$ we get then that $\mathcal{M}_{n} \preccurlyeq{ }_{I L} \mathcal{M}_{k}$, whence $\mathcal{M}_{n}, w \nvdash_{I L} \psi \rightarrow \chi$.

Our main theorem is then that no proper extension of IL displays the combination of useful properties established in Lemma 6. In other words, we are going to establish the following:

Theorem 1. Let $\mathcal{L}$ be an abstract intuitionistic logic. If $\mathrm{IL} \unlhd \mathcal{L}$ and $\mathcal{L}$ is invariant under asimulations, intuitionistically compact, and has the TUP, then $\mathrm{IL} \equiv \mathcal{L}$. 


\section{The Proof of Theorem 1}

Before we start with the proof, we need one more piece of notation. If $\mathcal{L}$ is an abstract intuitionistic logic, $\Theta$ a vocabulary, and $\Gamma \subseteq L(\Theta)$, then we let $\operatorname{Mod}_{\mathcal{L}}(\Theta, \Gamma)$ denote the class of pointed $\Theta$-models $(\mathcal{N}, u)$ such that for every $\phi \in \Gamma$ it is true that:

$$
\mathcal{N}, u \models_{\mathcal{L}} \phi .
$$

If $\Gamma=\{\phi\}$ for some $\phi \in L(\Theta)$ then instead of $\operatorname{Mod}_{\mathcal{L}}(\Theta, \Gamma)$ we simply write $\operatorname{Mod}_{\mathcal{L}}(\Theta, \phi)$.

We now start by establishing a couple of technical facts first:

Proposition 1. Let $\mathcal{L}$ be a almost strong $S$-closed abstract intuitionistic logic extending IL. Suppose that IL $\not \equiv \mathcal{L}$. Then, there are $\phi \in L\left(\Theta_{\phi}\right)$ and pointed $\Theta_{\phi}$-models $\left(\mathcal{M}_{1}, w_{1}\right),\left(\mathcal{M}_{2}, w_{2}\right)$ such that $T h_{I L}^{+}\left(\mathcal{M}_{1}, w_{1}\right) \subseteq T h_{I L}^{+}\left(\mathcal{M}_{2}, w_{2}\right)$ while $\mathcal{M}_{1}, w_{1} \models_{\mathcal{L}} \phi$ and $\mathcal{M}_{2}, w_{2} \not \nvdash_{\mathcal{L}} \phi$.

Proof. Suppose that for an arbitrary $\phi \in L\left(\Theta_{\phi}\right)$ we have shown that:

(i) $\operatorname{Mod}_{\mathcal{L}}\left(\Theta_{\phi}, \phi\right)=\bigcup_{(\mathcal{N}, u) \in \operatorname{Mod}_{\mathcal{L}}\left(\Theta_{\phi}, \phi\right)} \operatorname{Mod}_{\mathcal{L}}\left(\Theta_{\phi}, T h_{I L}^{+}(\mathcal{N}, u)\right)$,

Let $(\mathcal{N}, u) \in \operatorname{Mod}_{\mathcal{L}}\left(\Theta_{\phi}, \phi\right)$ be arbitrary. The above implies that every $\Theta_{\phi}$-model of $T h_{I L}^{+}(\mathcal{N}, u)$ must be a model of $\phi$. But then the theory $\left(T h_{I L}^{+}(\mathcal{N}, u),\{\phi\}\right)$ is $\mathcal{L}$ unsatisfiable. By the intuitionistic compactness of $\mathcal{L}$, for some finite $\Psi_{(\mathcal{N}, u)} \subseteq T h_{I L}^{+}(\mathcal{N}, u)$ (and we can pick a unique one using the Axiom of Choice), the theory $\left(\Psi_{(\mathcal{N}, u)},\{\phi\}\right)$ is $\mathcal{L}$-unsatisfiable. Hence, $\bigwedge \Psi_{(\mathcal{N}, u)}$ logically implies $\phi$ in $\mathcal{L}$. Then, given (i), we get that

(ii) $\operatorname{Mod}_{\mathcal{L}}\left(\Theta_{\phi}, \phi\right)=\bigcup_{(\mathcal{N}, u) \in \operatorname{Mod}_{\mathcal{L}}\left(\Theta_{\phi}, \phi\right)} \operatorname{Mod}_{\mathcal{L}}\left(\Theta_{\phi}, \Psi_{(\mathcal{N}, u)}\right)$.

However, this means that the theory $\left(\{\phi\},\left\{\bigwedge \Psi_{(\mathcal{N}, u)} \mid(\mathcal{N}, u) \in \operatorname{Mod}_{\mathcal{L}}\left(\Theta_{\phi}, \phi\right)\right\}\right)$ is $\mathcal{L}$ unsatisfiable and by (i), for some finite $\Gamma \subseteq\left\{\bigwedge \Psi_{(\mathcal{N}, u)} \mid(\mathcal{N}, u) \in \operatorname{Mod}_{\mathcal{L}}\left(\Theta_{\phi}, \phi\right)\right\}$, the theory $(\{\phi\}, \Gamma)$ is $\mathcal{L}$-unsatisfiable. This means that $\operatorname{Mod}_{\mathcal{L}}\left(\Theta_{\phi}, \phi\right) \subseteq \operatorname{Mod}_{\mathcal{L}}\left(\Theta_{\phi}, \bigvee \Gamma\right)$ So, using (ii), since clearly $\operatorname{Mod}_{\mathcal{L}}\left(\Theta_{\phi}, \bigvee \Gamma\right) \subseteq \bigcup_{(\mathcal{N}, u) \in \operatorname{Mod}_{\mathcal{L}}\left(\Theta_{\phi}, \phi\right)} \operatorname{Mod}_{\mathcal{L}}\left(\Theta_{\phi}, \Psi_{(\mathcal{N}, u)}\right)$, we get that:

(iii) $\operatorname{Mod}_{\mathcal{L}}\left(\Theta_{\phi}, \phi\right)=\operatorname{Mod}_{\mathcal{L}}\left(\Theta_{\phi}, \bigvee \Gamma\right)$.

Now, $\bigvee \Gamma$ is a perfectly good formula of $I L\left(\Theta_{\phi}\right)$ involving only finitary conjunctions and disjunctions. So we have shown that every $\phi \in L\left(\Theta_{\phi}\right)$ is just an intuitionistic $\Theta_{\phi}$-formula and hence that $\mathcal{L} \equiv \mathrm{IL}$ which is in contradiction with the hypothesis of the proposition.

Therefore, (i) must fail for at least one $\phi \in L\left(\Theta_{\phi}\right)$, and clearly, for this $\phi$ it can only fail if

$$
\bigcup_{(\mathcal{N}, u) \in \operatorname{Mod}_{\mathcal{L}}\left(\Theta_{\phi}, \phi\right)} \operatorname{Mod}_{\mathcal{L}}\left(\Theta_{\phi}, T h_{I L}^{+}(\mathcal{N}, u)\right) \nsubseteq \operatorname{Mod}_{\mathcal{L}}\left(\Theta_{\phi}, \phi\right) .
$$

But the latter means that for some pointed intuitionistic $\Theta_{\phi}$-model $\left(\mathcal{M}_{1}, w_{1}\right)$ such that $\mathcal{M}_{1}, w_{1} \models_{\mathcal{L}} \phi$ there is another $\Theta_{\phi}$-model $\left(\mathcal{M}_{2}, w_{2}\right)$ such that both $\mathcal{M}_{2}, w_{2} \nvdash_{\mathcal{L}} \phi$ and $T h_{I L}^{+}\left(\mathcal{M}_{1}, w_{1}\right)$ is satisfied at $\left(\mathcal{M}_{2}, w_{2}\right)$. The latter means, in turn, that we have $T h_{I L}^{+}\left(\mathcal{M}_{1}, w_{1}\right) \subseteq T h_{I L}^{+}\left(\mathcal{M}_{2}, w_{2}\right)$ as desired.

Assume $\mathcal{M}$ is a $\Theta$-model. We define that $\Theta_{\mathcal{M}}$ is $\Theta \cup\left\{q_{w}^{+}, q_{w}^{-} \mid w \in W\right\}$ such that $\Theta \cap\left\{q_{w}^{+}, q_{w}^{-} \mid w \in W\right\}=\varnothing$, and we define that $[\mathcal{M}]=(W, R,[V])$ is the $\Theta_{\mathcal{M}}$-model, such that $W$ and $R$ are just borrowed from $\mathcal{M}$ and $[V]$ coincides with $V$ on elements 
of $\Theta$, whereas for arbitrary $v, w \in W$ we set that $v \in[V]\left(q_{w}^{+}\right)$iff $w R v$ and $v \notin[V]\left(q_{w}^{-}\right)$ iff $v R w$.

Lemma 7. Let $\mathcal{L}$ be a bisimulation invariant abstract intuitionistic logic extending IL, let $(\mathcal{M}, w)$ be a pointed $\Theta$-model, and let $(\mathcal{N}, v)$ another pointed $\Theta_{\mathcal{M}_{w}^{u n} \text {-model. Assume }}$ that $T h_{\mathcal{L}}(\mathcal{N}, v)=T h_{\mathcal{L}}\left(\left[\mathcal{M}_{w}^{u n}\right], w\right)$. Then there exists an $\mathcal{L}$-embedding $f$ of $\left[\mathcal{M}_{w}^{u n}\right]$ into $\mathcal{N}$ such that $f(w)=v$.

Proof. Consider the relation $Z$ relating $w$ only to $v$, and every other element $\bar{u}_{k} \in W_{w}^{u n}$ to the worlds in $\mathcal{N}$ that $\mathcal{L}$-satisfy $T h_{\mathcal{L}}\left(\left[\mathcal{M}_{w}^{u n}\right], \bar{u}_{k}\right)$. We prove that such $Z$ is a total simulation from $\left(\left[\mathcal{M}_{w}^{u n}\right], w\right)$ to $(\mathcal{N}, v)$. Indeed, conditions (type) and (atom) are obviously satisfied. We treat condition (forth).

Assume that $\bar{v}_{k} Z v^{\prime}$ and that $\bar{v}_{k} R_{w}^{u n} \bar{v}_{n}$ (then $n \geq k$ ). By definition of $Z$ we have then $T h_{\mathcal{L}}\left(\mathcal{N}, v^{\prime}\right)=T h_{\mathcal{L}}\left(\left[\mathcal{M}_{w}^{u n}\right], \bar{v}_{k}\right)$, and also, since $w$ is the root of $\mathcal{M}_{w}^{u n}$, we have, by Lemma 5 and invariance of $\mathcal{L}$ under asimulations that

$$
T h_{\mathcal{L}}^{+}\left(\left[\mathcal{M}_{w}^{u n}\right], w\right) \subseteq T h_{\mathcal{L}}^{+}\left(\left[\mathcal{M}_{w}^{u n}\right], \bar{v}_{k}\right)=T h_{\mathcal{L}}^{+}\left(\mathcal{N}, v^{\prime}\right) .
$$

By definition of $\left[\mathcal{M}_{w}^{u n}\right]$ and closure of $\mathcal{L}$ w.r.t. intuitionistic implication, we have that the set:

$$
\Gamma_{\bar{v}_{n}}=\left\{q_{\bar{v}_{n}}^{+} \rightarrow \phi \mid \phi \in T h_{\mathcal{L}}^{+}\left(\left[\mathcal{M}_{w}^{u n}\right], \bar{v}_{n}\right)\right\} \cup\left\{\psi \rightarrow q_{\bar{v}_{n}}^{-} \mid \psi \in T h_{\mathcal{L}}^{-}\left(\left[\mathcal{M}_{w}^{u n}\right], \bar{v}_{n}\right)\right\}
$$

is a subset of $T h_{\mathcal{L}}^{+}\left(\left[\mathcal{M}_{w}^{u n}\right], w\right)$, thus also of $T h_{\mathcal{L}}^{+}\left(\mathcal{N}, v^{\prime}\right)$. We also know that, by $\bar{v}_{k} R_{w}^{u n} \bar{v}_{n}$, and by the fact that the theory $\left(\left\{q_{\bar{v}_{n}}^{+}\right\}\left\{q_{\bar{v}_{n}}^{-}\right\}\right)$is $\mathcal{L}$-satisfied at $\left(\left[\mathcal{M}_{w}^{u n}\right], \bar{v}_{n}\right)$, we have:

$$
\left[\mathcal{M}_{w}^{u n}\right], \bar{v}_{k} \not \nvdash_{\mathcal{L}} q_{\bar{v}_{n}}^{+} \rightarrow q_{\bar{v}_{n}}^{-} \text {. }
$$

Therefore, $\mathcal{N}, v^{\prime} \forall_{\mathcal{L}} q_{\bar{v}_{n}}^{+} \rightarrow q_{\bar{v}_{n}}^{-}$. So take some $v^{\prime \prime} \in U$ such that $v^{\prime} R^{\mathcal{N}} v^{\prime \prime}$ and $\left(\left\{q_{\bar{v}_{n}}^{+}\right\}\left\{q_{\bar{v}_{n}}^{-}\right\}\right)$is $\mathcal{L}$-satisfied at $\left(\mathcal{N}, v^{\prime \prime}\right)$. By $\Gamma_{\bar{v}_{n}} \subseteq T h_{\mathcal{L}}^{+}\left(\mathcal{N}, v^{\prime}\right)$, we also have that $\Gamma_{\bar{v}_{n}} \subseteq T h_{\mathcal{L}}^{+}\left(\mathcal{N}, v^{\prime \prime}\right)$, so that $T h_{\mathcal{L}}\left(\mathcal{N}, v^{\prime \prime}\right)=T h_{\mathcal{L}}\left(\left[\mathcal{M}_{w}^{u n}\right], \bar{v}_{n}\right)$, whence we get that $\bar{v}_{n} Z v^{\prime \prime}$ and condition forth is verified.

By Lemma 2, there must be then a homomorphism $f$ from $\left[\mathcal{M}_{w}^{u n}\right]$ to $\mathcal{N}$ such that $\bar{v}_{n} Z f\left(\bar{v}_{n}\right)$ for all $\bar{v}_{n} \in W_{w}^{u n}$, so, in particular, $w Z f(w)$ but by definition $Z$ only relates $w$ to $v$, hence $f(w)=v$.

For this $f$, we get condition (theories) immediately by definition of $Z$. To establish the injectivity of $f$ assume that $\bar{v}_{n}, \bar{u}_{k} \in W_{w}^{u n}$ are such that $\bar{v}_{n} \neq \bar{u}_{k}$. Then, by Lemma 1. either $\bar{v}_{n}$ is not an $R_{w}^{u n}$-successor of $\bar{u}_{k}$ or $\bar{u}_{k}$ is not an $R_{w}^{u n}$-successor of $\bar{v}_{n}$. If the first, $f\left(\bar{u}_{k}\right) \mathcal{L}$-satisfies $q_{\bar{u}_{k}}^{+}$in $\mathcal{N}$, while $f\left(\bar{v}_{n}\right)$ does not, so $f\left(\bar{u}_{k}\right) \neq f\left(\bar{v}_{n}\right)$. If the second, then a symmetric thing happens with $q_{\bar{v}_{n}}^{+}$.

Finally, if, for some $\bar{v}_{n}, \bar{u}_{k} \in W_{w}^{u n}, f\left(\bar{v}_{n}\right)$ is a successor of $f\left(\bar{u}_{k}\right)$ in $\mathcal{N}$, we must have that $\mathcal{N}, f\left(\bar{v}_{n}\right) \vDash_{\mathcal{L}} q_{\bar{u}_{k}}^{+}$, so that $\left[\mathcal{M}_{w}^{u n}\right], \bar{v}_{n} \vDash_{\mathcal{L}} q_{\bar{u}_{k}}^{+}$, which means by definition of the valuation of $q_{\bar{u}_{k}}^{+}$that $\bar{v}_{n}$ is an $R_{w}^{u n}$-successor of $\bar{u}_{k}$ in $\left[\mathcal{M}_{w}^{u n}\right]$ so that condition (s-hom) is verified as well.

Proposition 2. Let $(\mathcal{M}, w)$ be a pointed $\Theta$-model and let $\mathcal{L}$ be an abstract intuitionistic logic which is invariant under asimulations, intuitionistically compact, has TUP and extends IL. Then there is an $\mathcal{L}$-saturated pointed $\Theta$-model $(\mathcal{N}, s)$ such that $w \in U$ and $T h_{\mathcal{L}}(\mathcal{M}, w)=T h_{\mathcal{L}}(\mathcal{N}, w)$.

Proof. Consider $\left(\mathcal{M}_{w}^{u n}, w\right)$. By Lemma $13,\left(\mathcal{M}_{w}^{u n}, w\right)$ is bisimilar to $(\mathcal{M}, w)$, therefore, by Lemma 4 and invariance of $\mathcal{L}$ under asimulations, we get that $T h_{\mathcal{L}}(\mathcal{M}, w)=T h_{\mathcal{L}}\left(\mathcal{M}_{w}^{u n}, w\right)$. Consider then $\left[\mathcal{M}_{w}^{u n}\right]$ and assume $\bar{v}_{k} \in W_{w}^{u n}$. For a given $\bar{v}_{k} \in W_{w}^{u n}$, we will denote the 
family of all $L\left(\Theta_{\left[\mathcal{M}_{w}^{u n}\right]}\right)$-theories which are finitely $\mathcal{L}$-satisfiable in $\left[\mathcal{M}_{w}^{u n}\right]$ by successors of $\bar{v}_{k}$ by FinSat $\bar{v}_{k}$. For every $\bar{v}_{k} \in W_{w}^{u n}$ and every $(\Gamma, \Delta) \in$ FinSat $_{\bar{v}_{k}}$ we add to $\Theta_{\left[\mathcal{M}_{w}^{u n}\right]}$ a fresh pair of propositional letters $r_{\Gamma}^{+}$and $r_{\Delta}^{-}$. Call the resulting vocabulary $\Theta^{\prime}$. Consider next the following $L\left(\Theta^{\prime}\right)$-theory $\left(\Xi, T h_{\mathcal{L}}^{-}\left(\left[\mathcal{M}_{w}^{u n}\right], w\right)\right)$, where:

$$
\begin{aligned}
\Xi= & T h_{\mathcal{L}}^{+}\left(\left[\mathcal{M}_{w}^{u n}\right], w\right) \cup \\
& \cup\left\{r_{\Gamma}^{+} \rightarrow \phi, \psi \rightarrow r_{\Delta}^{-},\left(r_{\Gamma}^{+} \rightarrow r_{\Delta}^{-}\right) \rightarrow q_{\bar{v}_{k}}^{-} \mid(\Gamma, \Delta) \in \text { FinSat }_{\bar{v}_{k}}, \phi \in \Gamma, \psi \in \Delta, \bar{v}_{k} \in W_{w}^{u n}\right\},
\end{aligned}
$$

$\left(\Xi, T h_{\mathcal{L}}^{-}\left(\left[\mathcal{M}_{w}^{u n}\right], w\right)\right)$ is itself finitely $\mathcal{L}$-satisfiable, since given finite $\Xi_{0} \subseteq \Xi$ and $\Omega_{0} \subseteq T h_{\mathcal{L}}^{-}\left(\left[\mathcal{M}_{w}^{u n}\right], w\right)$, we know wlog that:

$$
\Xi_{0}=\Gamma \cup T\left(S_{\left(\Gamma_{1}, \Delta_{1}\right)}\right) \cup, \ldots, \cup T\left(S_{\left(\Gamma_{n}, \Delta_{n}\right)}\right),
$$

where $\Gamma$ is a finite subtheory of $T h_{\mathcal{L}}^{+}\left(\left[\mathcal{M}_{w}^{u n}\right], w\right),\left(\Gamma_{1}, \Delta_{1}\right) \in$ FinSat $_{\bar{v}_{k_{1}}}, \ldots,\left(\Gamma_{n}, \Delta_{n}\right) \in$ FinSat $_{\bar{v}_{k_{n}}}$, with $\bar{v}_{k_{1}}, \ldots, \bar{v}_{k_{n}} \in W_{w}^{u n}$, for every $1 \leq i \leq n S_{\left(\Gamma_{i}, \Delta_{i}\right)}$ is a finite subset of $\left(\Gamma_{i}, \Delta_{i}\right)$ and, again for every $1 \leq i \leq n$ :

$$
T\left(S_{\left(\Gamma_{i}, \Delta_{i}\right)}\right)=\left\{r_{\Gamma_{i}}^{+} \rightarrow \phi, \psi \rightarrow r_{\Delta_{i}}^{-},\left(r_{\Gamma_{i}}^{+} \rightarrow r_{\Delta_{i}}^{-}\right) \rightarrow q_{\bar{v}_{k_{i}}}^{-} \mid \phi, \psi \in S_{\left(\Gamma_{i}, \Delta_{i}\right)}\right\} .
$$

By definition of FinSat, every $S_{\left(\Gamma_{i}, \Delta_{i}\right)}$ for $1 \leq i \leq n$ will be $\mathcal{L}$-satisfied in some $v\left(S_{\left(\Gamma_{i}, \Delta_{i}\right)}\right) \in W_{w}^{u n}$ such that $\bar{v}_{k_{i}} R_{w}^{u n} v\left(S_{\left(\Gamma_{i}, \Delta_{i}\right)}\right)$. It is easy to see then, that every such $\left(\Xi_{0}, \Omega_{0}\right)$ will be $\mathcal{L}$-satisfied in the extension of $\left(\left[\mathcal{M}_{w}^{u n}\right], w\right)$, where for every $1 \leq i \leq n$ the propositional letters $r_{\Gamma_{i}}^{+}, r_{\Delta_{i}}^{-}$are identified with $q_{v\left(S_{\left(\Gamma_{i}, \Delta_{i}\right)}^{+}\right)}^{+} q_{v\left(S_{\left(\Gamma_{i}, \Delta_{i}\right)}\right)}^{-}$, respectively.

Therefore, by the intuitionistic compactness of $\mathcal{L},\left(\Xi, T h_{\mathcal{L}}^{-}\left(\left[\mathcal{M}_{w}^{u n}\right], w\right)\right)$ itself is $\mathcal{L}$ satisfiable. Let $\left(\mathcal{M}_{1}^{\prime}, w_{1}\right)$ be a pointed $\Theta^{\prime}$-model $\mathcal{L}$-satisfying $\left(\Xi, T h_{\mathcal{L}}^{-}\left(\left[\mathcal{M}_{w}^{u n}\right], w\right)\right)$, and we may assume that $w_{1}$ is the root of $\mathcal{M}_{1}^{\prime}$ (if not, throw away every world which is not accessible from $w_{1}$ in $\left.\mathcal{M}_{1}^{\prime}\right)$. Now, let $\mathcal{M}_{1}$ be the reduct of $\mathcal{M}_{1}^{\prime}$ to $\Theta_{\left[\mathcal{M}_{w}^{w n}\right]}$. We know that $\left(\mathcal{M}_{1}, w_{1}\right) \mathcal{L}$-satisfies $T h_{\mathcal{L}}\left(\left[\mathcal{M}_{w}^{u n}\right], w\right)$, therefore, by Lemma $1.3,\left(\left(\mathcal{M}_{1}\right)_{w_{1}}^{u n}, w_{1}\right)$ also $\mathcal{L}$-satisfies $T h_{\mathcal{L}}\left(\left[\mathcal{M}_{w}^{u n}\right], w\right)$ and by Lemma 7 there must be an embedding $f$ of $\left[\mathcal{M}_{w}^{u n}\right]$ into $\left(\mathcal{M}_{1}\right)_{w_{1}}^{u n}$ with $f(w)=w_{1}$. We now prove the following:

Claim. If $\bar{v}_{k} \in W_{w}^{u n}$ and a $L\left(\Theta_{\left[\mathcal{M}_{w}^{u n}\right]}\right)$-theory $(\Gamma, \Delta)$ is finitely $\mathcal{L}$-satisfiable in $\left[\mathcal{M}_{w}^{u n}\right]$ by successors of $\bar{v}_{k}$, then there is an $u \in\left(W_{1}\right)_{w_{1}}^{u n}$ such that $f\left(\bar{v}_{k}\right)\left(R_{1}\right)_{w_{1}}^{u n} u$ and $(\Gamma, \Delta)$ is $\mathcal{L}$-satisfied at $\left(\left(\mathcal{M}_{1}\right)_{w_{1}}^{u n}, u\right)$.

Indeed, we have $(\Gamma, \Delta) \in$ FinSat $_{\bar{v}_{k}}$, and we also have $\left[\mathcal{M}_{w}^{u n}\right], \bar{v}_{k} \forall_{\mathcal{L}} q_{\bar{v}_{k}}^{-}$, hence $\left(\mathcal{M}_{1}\right)_{w_{1}}^{u n}, f\left(\bar{v}_{k}\right) \nvdash_{\mathcal{L}} q_{\bar{v}_{k}}^{-}$. Since $\left(\mathcal{M}_{1}\right)_{w_{1}}^{u n}$ is an intuitionistic unravelling, $f\left(\bar{v}_{k}\right)$ is in fact some $\bar{u}_{r} \in\left(W_{1}\right)_{w_{1}}^{u n}$. By Lemma 1,4 we must have then $\mathcal{M}_{1}, u_{r} \nvdash_{\mathcal{L}} q_{\bar{v}_{k}}^{-}$, therefore, by Expansion, $\mathcal{M}_{1}^{\prime}, u_{r} \quad \forall_{\mathcal{L}} q_{\bar{v}_{k}}^{-}$. Since $w_{1}$ is the root of $\mathcal{M}_{1}^{\prime}$ and $\left(\Xi, T h_{\mathcal{L}}^{-}\left(\left[\mathcal{M}_{w}^{u n}\right], w\right)\right)$ is $\mathcal{L}$-satisfied at $\left(\mathcal{M}_{1}^{\prime}, w_{1}\right)$, we must also have $\mathcal{M}_{1}^{\prime}, u_{r} \models_{\mathcal{L}} \Xi$, and, in particular, $\mathcal{M}_{1}^{\prime}, u_{r} \models_{\mathcal{L}}\left(r_{\Gamma}^{+} \rightarrow r_{\Delta}^{-}\right) \rightarrow q_{\bar{v}_{k}}^{-}$. Therefore, there must be a successor $u^{\prime}$ to $u_{r}$ in $\mathcal{M}_{1}^{\prime}$ such that $\left(\left\{r_{\Gamma}^{+}\right\},\left\{r_{\Delta}^{-}\right\}\right)$is $\mathcal{L}$-satisfied at $\left(\mathcal{M}_{1}^{\prime}, u^{\prime}\right)$. Since we will also have $\mathcal{M}_{1}^{\prime}, u^{\prime} \models_{\mathcal{L}} \Xi$, this means that $(\Gamma, \Delta)$ will be $\mathcal{L}$-satisfied at $\left(\mathcal{M}_{1}^{\prime}, u^{\prime}\right)$ as well. Again by Expansion, we get that $(\Gamma, \Delta)$ will be $\mathcal{L}$-satisfied at $\left(\mathcal{M}_{1}, u^{\prime}\right)$ and further, by Lemma 1,4, that $(\Gamma, \Delta)$ will be $\mathcal{L}$-satisfied at $\left(\left(\mathcal{M}_{1}\right)_{w_{1}}^{u n},\left(\bar{u}_{r}, u^{\prime}\right)\right)$. By $u_{r} R_{1} u^{\prime}$, we have that $\left(\bar{u}_{r}, u^{\prime}\right) \in\left(W_{1}\right)_{w_{1}}^{u n}$ and $\bar{u}_{r}\left(R_{1}\right)_{w_{1}}^{u n}\left(\bar{u}_{r}, u^{\prime}\right)$. Therefore, setting $u:=\left(\bar{u}_{r}, u^{\prime}\right)$, we get our Claim verified.

Observe further, that by the remark after Definition 4 , the existence of the abovedefined embedding $f$ means that we may assume that $\left[\mathcal{M}_{w}^{u n}\right] \preccurlyeq \mathcal{L}\left(\mathcal{M}_{1}\right)_{w_{1}}^{u n}$ and identify

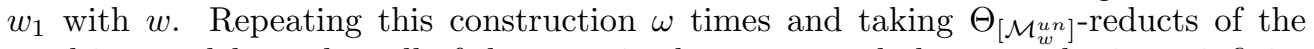
resulting models so that all of them are in the same vocabulary, we obtain an infinite 
chain of $\mathcal{L}$-elementary submodels of the following form:

$$
\left[\mathcal{M}_{w}^{u n}\right] \preccurlyeq \mathcal{L}\left(\mathcal{M}_{1}\right)_{w}^{u n} \preccurlyeq \mathcal{L}, \ldots, \preccurlyeq \mathcal{L}\left(\mathcal{M}_{n}\right)_{w}^{u n} \preccurlyeq \mathcal{L}, \ldots,
$$

with $w$ being the root of every model in the chain. To simplify notation and achieve the uniformity of subscripts, we rename $\left[\mathcal{M}_{w}^{u n}\right]$ as $\mathcal{N}_{0}$, and for $i \geq 1$, rename $\left(\mathcal{M}_{i}\right)_{w}^{u n}$ as $\mathcal{N}_{i}$. We consider then $\bigcup_{i \in \omega} \mathcal{N}_{i}$. Since $\mathcal{L}$ has TUP, we know that for every $j \in \omega$ we have

$$
\mathcal{N}_{j} \preccurlyeq \mathcal{L} \bigcup_{i \in \omega} \mathcal{N}_{i}
$$

We claim that $\bigcup_{i \in \omega} \mathcal{N}_{i}$ is an $\mathcal{L}$-saturated $\Theta_{\left[\mathcal{M}_{w}^{u n}\right]}$-model. Indeed, assume that $(\Gamma, \Delta)$ is an $L\left(\Theta_{\left[\mathcal{M}_{w}^{u n}\right]}^{u}\right)$-theory which is finitely $\mathcal{L}$-satisfiable among successors of some $v$ in $\bigcup_{i \in \omega} \mathcal{N}_{i}$. Then for any finite $\left(\Gamma_{0}, \Delta_{0}\right) \subseteq(\Gamma, \Delta)$ we will have

$$
\bigcup_{i \in \omega} \mathcal{N}_{i}, v \not \forall_{\mathcal{L}} \bigwedge \Gamma_{0} \rightarrow \bigvee \Delta_{0}
$$

Then choose $j \in \omega$ such that $\mathcal{N}_{j}$ is the first model in the chain, where $v$ occurs. By (11), we will have:

$$
\mathcal{N}_{j}, v \forall_{\mathcal{L}} \bigwedge \Gamma_{0} \rightarrow \bigvee \Delta_{0}
$$

for any finite $\left(\Gamma_{0}, \Delta_{0}\right) \subseteq(\Gamma, \Delta)$. Therefore, $(\Gamma, \Delta)$ will be finitely $\mathcal{L}$-satisfiable in $\mathcal{N}_{j}$ by successors of $v$ and by the respective version of our Claim above, this means that there is a successor $v^{\prime}$ to $v$ in $\mathcal{N}_{j+1}$ such that $(\Gamma, \Delta)$ is $\mathcal{L}$-satisfied at $\left(\mathcal{N}_{j+1}, v^{\prime}\right)$. But then, again by (11), we get that $(\Gamma, \Delta)$ is also $\mathcal{L}$-satisfied at $\left(\bigcup_{i \in \omega} \mathcal{N}_{i}, v^{\prime}\right)$.

Since $\bigcup_{i \in \omega} \mathcal{N}_{i}$ is therefore shown to be an $\mathcal{L}$-saturated $\Theta_{\left[\mathcal{M}_{w}^{\text {un }}\right]}$-model, then setting $\mathcal{N}$ to be $\Theta$-reduct of $\bigcup_{i \in \omega} \mathcal{N}_{i}$, we immediately get that $\mathcal{N}$ is $\mathcal{L}$-saturated. Also, by (11) and the fact that $\mathcal{M}_{w}^{u n}$ is the $\Theta$-reduct of $\left[\mathcal{M}_{w}^{u n}\right]$ we get that:

$$
T h_{\mathcal{L}}\left(\mathcal{M}_{w}^{u n}, w\right)=T h_{\mathcal{L}}(\mathcal{N}, w) .
$$

Therefore, by Lemma 1,4, we get that $T h_{\mathcal{L}}(\mathcal{M}, w)=T h_{\mathcal{L}}(\mathcal{N}, w)$ and thus we are done.

We are now in a position to prove Theorem 1. Indeed, assume the hypothesis of the theorem, and assume, for contradiction, that $\mathcal{L} \not \equiv \mathrm{IL}$. By Proposition 1 there must be $\phi \in L\left(\Theta_{\phi}\right)$ and pointed intuitionistic $\Theta_{\phi}$-models $\left(\mathcal{M}_{1}, w_{1}\right),\left(\mathcal{M}_{2}, w_{2}\right)$ such that $T h_{I L}^{+}\left(\mathcal{M}_{1}, w_{1}\right) \subseteq T h_{I L}^{+}\left(\mathcal{M}_{2}, w_{2}\right)$ while $\mathcal{M}_{1}, w_{1} \models_{\mathcal{L}} \phi$ and $\mathcal{M}_{2}, w_{2} \forall_{\mathcal{L}} \phi$. By Proposition 2, take $\mathcal{L}$-saturated $\Theta_{\phi}$-models $\mathcal{N}_{1}$ and $\mathcal{N}_{2}$ such that $w_{i} \in U_{i}$ and $T h_{\mathcal{L}}\left(\mathcal{M}_{i}, w_{i}\right)=T h_{\mathcal{L}}\left(\mathcal{N}_{i}, w_{i}\right)$ for $i \in\{0,1\}$. We will have then, of course, that $\mathcal{N}_{1}, w_{1} \models_{\mathcal{L}} \phi$, but $\mathcal{N}_{2}, w_{2} \nvdash_{\mathcal{L}} \phi$. On the other hand, we will still have $T h_{I L}^{+}\left(\mathcal{N}_{1}, w_{1}\right) \subseteq T h_{I L}^{+}\left(\mathcal{N}_{2}, w_{2}\right)$, whence by Corollary 1 there must be an asimulation $A$ from $\left(\mathcal{N}_{1}, w_{1}\right)$ to $\left(\mathcal{N}_{2}, w_{2}\right)$, but then, since $\mathcal{L}$ is invariant under asimulations, we must also have $T h_{\mathcal{L}}^{+}\left(\mathcal{N}_{1}, w_{1}\right) \subseteq T h_{\mathcal{L}}^{+}\left(\mathcal{N}_{2}, w_{2}\right)$. Now, since $\phi \in T h_{\mathcal{L}}^{+}\left(\mathcal{N}_{1}, w_{1}\right)$, we can see that $\phi \in T h_{\mathcal{L}}^{+}\left(\mathcal{N}_{2}, w_{2}\right)$, so that $\mathcal{N}_{2}, w_{2} \models_{\mathcal{L}} \phi$, which is a contradiction.

\section{A topological approach to the main theorem}

There is a topological reading of our result. This sort of take on Lindström theorems has been explored by some authors, in particular, Caicedo (see [5]). One of the fundamental interests of such an approach is that logical compactness properties become topological compactness properties. To see this, for each vocabulary $\Theta$, we must first 
define a topology (to be called the intuitionistic topology for $\Theta$-indeed, the reference to $\Theta$ will be dropped when the context makes it clear that some $\Theta$ has been fixed) on the the space of all intuitionistic pointed $\Theta$-models (call it $S$ for the purposes of this section). Abstract logics will then roughly correspond to topologies on such space. We will assume that the reader is familiar with the basic notions of general topology that can be acquired in a place like [13.

We start by introducing a closed base $B$ for our topology. The elements of $B$ have the form $\operatorname{Mod}(\phi)$ for some formula $\phi$ in the usual language $\{\rightarrow, \wedge, \vee, \perp, \top\}$ of intuitionistic propositional logic. B is a closed base for a topology on $S$ because for any two $A, B \in \mathrm{B}$ there is $W \in \mathrm{B}$ such that $A \cup B=W$ : given $\operatorname{Mod}(\phi)$ and $\operatorname{Mod}(\psi)$, consider $\operatorname{Mod}(\phi \vee \psi)$. The intuitionistic topology is just the topology induced by this base, that is, take as closed collections arbitrary intersections of elements of B.

Now we may observe that we ended up with a topology where the closed collections have the form $\operatorname{Mod}(T)$ for some intuitionistic set of formulas $T$. Denote $2^{I L(\Theta)}$ with $\wp(F m l a)$. In particular, given a subclass $A$ of the space, if we denote by $T h_{I L}^{+}(A)$ the collection of all intuitionistic formulas holding in every member of $A$, then $A^{-}$, the closure of $A$ in our topology, is just $\bigcap_{\substack{T \in \mathcal{B}(F m l a) \\ A \subseteq M \text { od }(T)}} \operatorname{Mod}(T)=\operatorname{Mod}\left(T h_{I L}^{+}(A)\right)$.

The space is not normal (i.e., it is not the case that for any two disjoint closed classes $A, B$ there exist disjoint open classes $U, V$ containing $A$ and $B$ respectively), for let $\operatorname{Mod}(T) \cap \operatorname{Mod}(U)=\emptyset$ for two intuitionistic theories such that $\operatorname{Mod}(T) \neq \emptyset$ and $\operatorname{Mod}(U) \neq \emptyset$. Moreover, suppose that $\operatorname{Mod}(T) \subseteq S \backslash \operatorname{Mod}\left(T^{\prime}\right)$, and $\operatorname{Mod}(U) \subseteq S \backslash \operatorname{Mod}\left(U^{\prime}\right)$ for some intuitionistic theories $T^{\prime}, U^{\prime}$. Now take any structure $(\mathcal{M}, w) \in S \backslash \operatorname{Mod}\left(T^{\prime}\right)$ and $(\mathcal{N}, v) \in S \backslash \operatorname{Mod}\left(U^{\prime}\right)$, but this means that for some $\phi \in T^{\prime}$ and $\psi \in U^{\prime}$ we have that $\phi$ and $\psi$ fail at $(\mathcal{M}, w)$ and $(\mathcal{N}, v)$ respectively, so neither $\phi$ nor $\psi$ are theorems of intuitionistic logic. But intuitionistic logic has the disjunction property $(\vdash \phi \vee \psi$ only if either $\vdash \phi$ or $\vdash \psi$ ), so in fact there is some $\left(\mathcal{M}^{\prime}, w^{\prime}\right) \in S$ such that $\phi \vee \psi$ fails, which implies that $\left(\mathcal{M}^{\prime}, w^{\prime}\right) \in\left(S \backslash \operatorname{Mod}\left(T^{\prime}\right)\right) \cap\left(S \backslash \operatorname{Mod}\left(U^{\prime}\right)\right)$.

The space is not regular (i.e., it is not the case that closed classes and exterior points may be separated by disjoint open classes). Take any $\operatorname{Mod}\left(T_{0}\right) \neq \emptyset$ and $(\mathcal{M}, w) \notin \operatorname{Mod}\left(T_{0}\right)$, i.e., $\mathcal{M}, w \nvdash_{I L} \phi$ for some $\phi \in T_{0}$. Consider further arbitrary open classes $S \backslash \operatorname{Mod}\left(T_{1}\right)$ and $S \backslash \operatorname{Mod}\left(T_{2}\right)$ such that $\operatorname{Mod}\left(T_{0}\right) \subseteq S \backslash \operatorname{Mod}\left(T_{1}\right)$ and $(\mathcal{M}, w) \in S \backslash \operatorname{Mod}\left(T_{2}\right)$. Hence, taking $(\mathcal{N}, v) \in \operatorname{Mod}\left(T_{0}\right)$, it must be in $S \backslash \operatorname{Mod}\left(T_{1}\right)$. So there are $\psi \in T_{1}$ and $\theta \in T_{2}$ such that $\mathcal{N}, v \forall_{I L} \psi$ and $\mathcal{M}, w \forall_{I L} \theta$. By the disjunction property, we have $(\mathcal{C}, u)$ falsifying both $\psi$ and $\theta$, hence the intersection $\left(S \backslash \operatorname{Mod}\left(T_{1}\right)\right) \cap\left(S \backslash \operatorname{Mod}\left(T_{2}\right)\right)$ cannot be empty.

The space is compact because each family of closed sets which has the finite intersection property has a non-empty intersection (this is just the content of the compactness theorem in intuitionistic logic - hence Rasiowa's name for this theorem), which is equivalent to saying that every covering of the space can be reduced to a finite subcovering.

Moreover, the space is connected. To see this we again use the disjunction property of intuitionistic logic. For suppose that $S=A \cup B$ for separated subsets $A, B \neq \emptyset$. But then $\operatorname{Mod}\left(T h_{I L}^{+}(A)\right) \cap B=\emptyset$ and $\operatorname{Mod}\left(T h_{I L}^{+}(B)\right) \cap A=\emptyset$. Now take $(\mathcal{M}, w) \in A$, so it must be the case that some $\phi \in T h_{I L}^{+}(A)$ fails in $(\mathcal{M}, w)$. On the other hand, taking $(\mathcal{N}, v) \in B$, we obtain an $(\mathcal{N}, v)$ where some $\psi \in T h_{I L}^{+}(B)$ fails. By the disjunction property, there is a structure $\left(\mathcal{M}^{\prime}, w^{\prime}\right)$ where both $\phi$ and $\psi$ fail, which means that $\left(\mathcal{M}^{\prime}, w^{\prime}\right)$ is in neither the closure of $A$ nor the closure of $B$, which is impossible given that $S=A \cup B$. 
The space is not Hausdorff because given two models satisfying exactly the same intuitionistic formulas (that is, they belong to exactly the same closed classes of the topology) but which are not isomorphic, so they are distinct, we have a violation of the definition of a Hausdorff space: given two distinct points in the space we can find disjoint neighborhoods of each of them. In fact, we get with this example that the space is not $T_{1}$ : there are distinct points $x, y$ such that for every open classes $A, B$ of the intuitionistic topology, $x \in A$ only if $y \in A$ and $y \in B$ only if $x \in B$. Even worse, the space is not even $T_{0}$ : by the same example we have got points $x, y$ such that there is no neighborhood of $x$ where $y$ doesn't belong. Naturally, the latter observation suffices to refute the earlier properties.

Now we look at a related topology that plays an important role in our main result. Consider the collection SB* containing all collections of the form $S \backslash \operatorname{Mod}(\phi, \perp)$ and $S \backslash \operatorname{Mod}(\top, \psi)$ for all intutionistic formulas $\phi$ and $\psi$. This forms an open subbase for a topology on $S$. The open base $\mathrm{B}^{*}$ generated from $\mathrm{SB}^{*}$ is just all classes of the form $\operatorname{Mod}(\phi, \psi)$ for intuitionistic formulas $\phi$ and $\psi$ (this collection simply contains all finite intersections of members of $\mathrm{SB}^{*}$ ). Open classes in this topology are of the form $\bigcup_{\phi \in \Phi, \psi \in \Psi} \operatorname{Mod}(\phi, \psi)$ for some collections $\Phi, \Psi$ of formulas.

A topological space is said to be strongly $S$-closed if every family of open sets with the finite intersection property has a non-empty intersection 17. Moreover, we will say that a space is almost strongly S-closed if every family of basic open sets with the finite intersection property has a non-empty intersection. The topology is almost strongly Sclosed this follows from the fact that every cover of $S$ by members of the subbase has a finite subcover. The latter comes from the intuitionistic compactness of intuitionistic logic. Moreover, the topology is uniform, we can obtain it from what we will call (borrowing from [5]) the canonical uniformity, namely the uniformity generated by the base formed by the subclass $U \subseteq S \times S$ containing:

$$
U_{\Phi}=\left\{((\mathcal{M}, w),(\mathcal{N}, v)) \mid \mathcal{M}, w \models_{I L} \phi \text { iff } \mathcal{N}, v \models_{I L} \phi, \phi \in \Phi\right\}
$$

for each finite collection $\Phi$ of formulas. Now, the canonical uniformity generates the previously defined topology of intuitionistic theories. To see this, first note that $U_{\Phi}[(\mathcal{M}, w)]=\left\{(\mathcal{N}, v) \mid((\mathcal{M}, w),(\mathcal{N}, v)) \in U_{\Phi}\right\}$ coincides with

$$
\left(S \backslash\left\{(\mathcal{N}, v) \mid \mathcal{N}, v \models_{I L}\left(\bigvee \Phi^{-}, \perp\right)\right\}\right) \cap\left(S \backslash\left\{(\mathcal{N}, v) \mid \mathcal{N}, v \models_{I L}\left(\top, \bigwedge \Phi^{+}\right)\right\}\right)
$$

where $\Phi^{+}=\left\{\phi \in \Phi \mid \mathcal{M}, w \models_{I L} \phi\right\}$ while $\Phi^{-}=\left\{\phi \in \Phi \mid \mathcal{M}, w \forall_{I L} \phi\right\}$. Now, given an open class $\bigcup_{\phi \in \Phi, \psi \in \Psi} \operatorname{Mod}(\phi, \psi)$, if $(\mathcal{M}, w) \in \bigcup_{\phi \in \Phi, \psi \in \Psi} \operatorname{Mod}(\phi, \psi)$, then for some $\phi, \psi$, we have that $(\mathcal{M}, w) \in \operatorname{Mod}(\phi, \psi)$. But then $U_{\{\phi, \psi\}}[(\mathcal{M}, w)] \subseteq \operatorname{Mod}(\phi, \psi)$. Hence, open classes of the previously considered topology are open in the topology of the canonical uniformity. Conversely, if $O$ is an open class of the topology of the canonical uniformity, we can see that $O=\bigcup_{\substack{(\mathcal{M}, w) \in \mathcal{O},(\mathcal{M}, w) \leq 0, U_{\Phi} \in U}} U_{\Phi}[(\mathcal{M}, w)]$. But the latter is a union of open classes in the previously considered topology, as we have observed above.

Another space that may be interesting to explore is the quotient space obtained from the following equivalence relation on $S: x \approx y$ iff $x, y$ belong to exactly the same closed classes of the intuitionistic topology. Now consider the quotient topology for the natural projection $\pi: S \longrightarrow S \backslash \approx$. This topology takes as the closed classes all those subclasses $U$ of $S$ such that $\pi^{-1}[U]$ is closed in the intuitionistic topology. So 
the closed classes in this topology have the form $\{[(\mathcal{M}, w)] \mid(\mathcal{M}, w) \in \operatorname{Mod}(T)\}$ for some intuitionistic set of formulas $T$.

The quotient space is $T_{0}$ : for if $[(\mathcal{M}, w)] \neq[(\mathcal{N}, v)]$ then we have some $\phi$ such that $(\mathcal{M}, w) \in \operatorname{Mod}(\phi)$ while $(\mathcal{N}, v) \notin \operatorname{Mod}(\phi)$, so $[(\mathcal{M}, w)] \in\{[(\mathcal{M}, w)] \mid(\mathcal{M}, w) \in \operatorname{Mod}(\phi)\}$ whereas $[(\mathcal{N}, v)] \notin\{[(\mathcal{N}, v)] \mid(\mathcal{N}, v) \in \operatorname{Mod}(\phi)\}$, so there is a neighborhood of one of the points not containing the other.

On the other hand, the space is not Hausdorff: take $[(\mathcal{M}, w)] \neq[(\mathcal{N}, v)]$, and notice that for every open classes $U, V$ such that $[(\mathcal{M}, w)] \in U$ and $[(\mathcal{N}, v)] \in V$ we have that, for some sets of intuitionistic formulas $T, O$, we have that $U=(S \backslash \approx) \backslash\{[(\mathcal{C}, u)] \mid(\mathcal{C}, u) \in \operatorname{Mod}(T)\}$ and $V=(S \backslash \approx) \backslash\{[(\mathcal{C}, u)] \mid(\mathcal{C}, u) \in \operatorname{Mod}(O)\}$. So $(\mathcal{M}, w) \notin \operatorname{Mod}(\phi)$ and $(\mathcal{N}, v) \notin \operatorname{Mod}(\psi)$ for some $\phi \in T$ and $\psi \in U$, so using the disjunction property, we get some $\left(\mathcal{M}^{\prime}, w^{\prime}\right)$ such that $\left(\mathcal{M}^{\prime}, w^{\prime}\right) \notin \operatorname{Mod}(\phi \vee \psi)$, which means that $\left[\left(\mathcal{M}^{\prime}, w^{\prime}\right)\right] \in U \cap V$.

Finally, the main theorem of this paper may be read as: for any vocabulary, the intuitionistic topology is the finest topology such that (i) its closed sets are closed under asimulations, (ii) it has the Tarski-Union property and (iii) the subbase of the topology of the canonical uniformity is such that every open cover of the space by members of the subbase has a finite subcover.

\section{Conclusion}

We have succeeded in establishing a Lindström characterization of intuitionistic propositional logic over intuitionistic models involving the properties of intuitionistic compactness, the TUP and preservation under asimulations. In future work we will show how this characterization can be extended to the case of predicate intuitionistic logic. Our work, of course, opens a world of new questions. A particularly immediate one is to find some other combination of interesting model theoretic properties that implies the three mentioned properties and would therefore give us a new intuitionistic Lindström theorem.

On the other hand, it would be nice to work out the details of what is the algebraic content of our result via duality theory. Pointed intuitionistic models are dual to Heyting matrices (that is, pairs formed by a Heyting algebra and a filter on such algebra), so the work reduces to investigate the algebraic counterparts of the TUP and the preservation under asimulations.

\section{ACKNOWLEDGMENTS}

We are grateful to the anonymous referee and the editor of this journal for their time and effort on this paper. This article is a testimony to the eternal indestructible friendship between the Cuban and Soviet peoples.

Guillermo Badia is supported by the project I 1923-N25 of the Austrian Science Fund (FWF). Grigory Olkhovikov is supported by Deutsche Forschungsgemeinschaft (DFG), project WA 936/11-1.

\section{REFERENCES}

[1] J. Barwise. Axioms for abstract model theory. Annals of Mathematical Logic 7: 221-265 (1974).

[2] J. Barwise, S. Feferman, eds.. Model-Theoretic Logics, Springer-Verlag (1985).

[3] P. Blackburn, M. de Rijke, Y. Venema. Modal Logic, CUP (2001).

[4] A. Chagrov and M. Zakharyaschev. Modal Logic, Clarendon Press (1997). 
[5] X. Caicedo Continuous operations on spaces of structures. In: Quantifiers: Logics, Models and Computation, Synthese Library, Vol. 248, pp. 263-296 (1995).

[6] M. De Rijke. A Lindström Theorem for Modal Logic. In: Modal Logic and Process Algebra. Lecture Notes 53, CSLI Publications, Stanford (1995).

[7] J. Dontchev. Contra-continuous Functions and Strongly S-closed Spaces. Internat. J. Math. Sci., 19: 303-310 (1996).

[8] S. Enqvist. A General Lindström Theorem for Some Normal Modal Logics. Logica Universalis, 7(2): 233-264 (2013)

[9] S. Feferman. Two notes on abstract model theory. I. Properties invariant on the range of definable relations between structures. Fundamenta Mathematicae, 82: 153-165 (1974) .

[10] J. Flum. First-order logic and its extensions. In: Proceedings of the International Summer Institute and Logic Colloquium, Kiel 1974, Springer-Verlag, pp. 248-310 (1975).

[11] D. Gabbay and L. Maksimova. Interpolation and Definability: Modal and Intuitionistic Logics, OUP (2005).

[12] M. García-Matos and J. Väänänen. Abstract Model Theory as a Framework for Universal Logic. In: Logica Universalis: Towards a General Theory of Logic, Jean-Yves Beziau (ed.), Birkhäuser (2005).

[13] J. L. Kelley. General Topology, Van Nostrand (1967).

[14] N. Kurtonina and M. de Rijke. Simulating without negation. Journal of Logic and Computation, 7 (4): 501-522 (1997).

[15] P. Lindström. On extensions of elementary logic. Theoria 35: 1-11 (1969).

[16] G. K. Olkhovikov. Model-theoretic Characterization of Intuitionistic Propositional Formulas. The Review of Symbolic Logic, 6 (2): 348-365 (2013).

[17] G. K. Olkhovikov. Model-theoretic characterization of intuitionistic predicate formulas. Journal of Logic and Computation, 24: 809-829 (2014).

[18] G. K. Olkhovikov. Expressive power of basic modal intuitionistic logic as a fragment of classical FOL. Journal of Applied Logic, http://dx.doi.org/10.1016/j.jal.2016.11.036.

[19] G. K. Olkhovikov. On generalized Van-Benthem-type characterizations. Annals of Pure and Applied Logic , 168 (9): 1643-1691 (2017).

[20] R. Piro and M. Otto. A Lindström Characterisation of the Guarded Fragment and of Modal Logic With a Global Modality. In: Advances in Modal Logic 7, AiML 2008, C. Areces and R. Goldblatt (eds), pp. 273-288.

[21] J. van Benthem. A new modal Lindström theorem. Logica Universalis 1 (1):125-138 (2007).

Department of Knowledge-Based Mathematical Systems, Johannes Kepler University, Linz, Austria

School of Historical and Philosophical Inquiry, University of Queensland, Brisbane, Australia

E-mail address: guillebadia89@gmail.com

Department of Philosophy, Ruhr University Bochum, Bochum, Germany

E-mail address: grigory.olkhovikov@rub.de 